Published in final edited form as:

Yang, S. J., Rothe, R., Kirchhecker, S., Esposito, D., Antonietti, M., Gojzewski, H. \& Fechler, N. (2015). A Sustainable Synthesis Alternative for IL-derived N-doped Carbons: Bio-based-Imidazolium Compounds. Carbon, 94, 641-645. doi:10.1016/j.carbon.2015.07.034.

\title{
A Sustainable Synthesis Alternative for IL-derived N-doped Carbons: Bio-based-Imidazolium Compounds
}

\begin{abstract}
Herein we report a facile and scalable synthesis of highly nitrogen-doped porous carbon materials with tunable morphology. Bio-based imidazolium derivatives made from natural amino acids and dioxo-derivatives are employed as precursors, exhibiting an analogous behavior during carbonization to classical ionic liquids. Utilization of systematically controlled salt templating methods yields nitrogen-doped carbon materials with high surface areas of up to $2650 \mathrm{~m}^{2} \mathrm{~g}^{-1}$ and morphology-engineered structures such as monolithic or highly extended, sheet-like carbons. We believe that the presented approach represents an alternative and sustainable platform towards the rational design of carbon materials possessing controlled nanoporosity and functionality.
\end{abstract}




\title{
A Sustainable Synthesis Alternative for IL-derived N-doped Carbons: Bio-based-Imidazolium Compounds
}

Seung Jae Yang, Regina Rothe, Sarah Kirchhecker, Davide Esposito, Markus Antonietti, Hubert Gojzewski, Nina Fechler*

\author{
Max Planck Institute of Colloids and Interfaces, Department of Colloid Chemistry, MPI \\ Campus Golm, Am Mühlenberg 1, 14476 Potsdam, Germany
}

\begin{abstract}
Herein we report a facile and scalable synthesis of highly nitrogen-doped porous carbon materials with tunable morphology. Bio-based imidazolium derivatives made from natural amino acids and dioxo-derivatives are employed as precursors, exhibiting an analogous behavior during carbonization to classical ionic liquids. Utilization of systematically controlled salt templating methods yields nitrogen-doped carbon materials with high surface areas of up to $2650 \mathrm{~m}^{2} \mathrm{~g}^{-1}$ and morphology-engineered structures such as monolithic or highly extended, sheet-like carbons. We believe that the presented approach represents an alternative and sustainable platform towards the rational design of carbon materials possessing controlled nanoporosity and functionality.
\end{abstract}

Recent experiments reveal more and more "designer carbons", i.e. functional carbon materials which possess highly favorable, diverse and especially tunable properties. ${ }^{[1,2,3]}$ Such carbons can be found in many applications and devices such as electrode materials for energy storage and conversion, as filters with adjustable adsorption properties and selectivity, and for thermal isolation and electricity generation. ${ }^{[4,5,6]}$ With regard to applications in every-day-life, it is equally important to satisfy criteria of broad and scalable availability while costs and environmental impacts are to be kept at a minimum.

*Corresponding author:

Phone: +49 3315679569

E-mail: Nina.Fechler@mpikg.mpg.de (Nina Fechler) 
Recently, ionic liquids have been discovered as promising new class of carbon precursors as they possess several advantageous properties: the liquid state enables facile shaping and processing, heteroatoms can be introduced by the choice of ionic liquid type, and vapor pressure is negligible eventually making special synthesis equipment obsolete. ${ }^{[7,}{ }^{8]}$ Additionally, due to their liquid character, they can be easily mixed or infiltrated with additional substances. However, mostly because of the challenging synthetic and purification processes, common ionic liquids are still rather expensive. Therefore, the need for reduced costs for carbon materialization of ionic liquids has been established as one of the main challenges in this field. ${ }^{[9]}$ Besides existing works on protic ionic liquids ${ }^{[10]}$ with regard to price and improved sustainability, we recently presented the convenient synthesis of bifunctional imidazolium compounds using natural amino acids as green starting materials. ${ }^{[1]}$ The synthesis of such imidazoliums can be entirely performed according to the rules of green chemistry at reduced costs, while control over the specific $N$-substitution of the imidazole moiety can be achieved by selection of different amino acids.

In the present paper these "bio-imidazoliums" (bio- $\mathrm{Im}$ ) are applied for the synthesis of functional heteroatom-doped carbons. These precursors can be designed and mixed in an individual fashion so that special functional groups can tune the final carbon properties, such as nitrogen content and patterning of functionality. High surface areas of $2658 \mathrm{~m}^{2} \mathrm{~g}^{-1}$ could be achieved via the salt templating approach, without any consecutive activation. The choice of salt also allowed the synthesis of sheet-like, two-dimensional carbon nanostructures. In addition, the combination of bio-Im and salt leads to a fourfold increase of yield with respect to carbonization of the pure compound. We believe that this approach is highly attractive for applications where high amounts of functional carbons with controlled pore structure and functionality are needed.

The synthesis of functional carbons from bio-imidazoliums (bio-Im) is representatively delineated by using two different imidazoliums, constructed from two different amino acids (glycine and phenylalanine), yet bearing the same basic structure to enable direct comparison of materials (Figure $1 \mathbf{a}, \mathbf{b}$ ). Both possess a central imidazolium core with two carboxylic acid substituents, however, bio-Im 2 contains two additional phenyl-groups. Recently, we showed that the hydrothermal treatment of amino acid-derived imidazoliums leads to the formation of "classical" ionic liquids (IL) via decarboxylation at the $\alpha$-amino acid 
carbons contained in the bio-Im. ${ }^{[12]}$ In a similar way, we surmised that during the high temperature treatment the bio-Im may undergo a similar decarboxylative process leading to a bio-derived IL as transient species. Therefore, Bio-Ims can be considered as a source of “masked-IL".

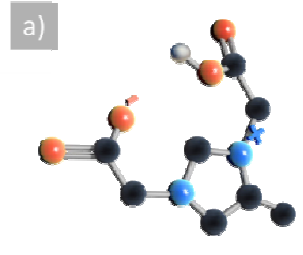

Bio-Im 1
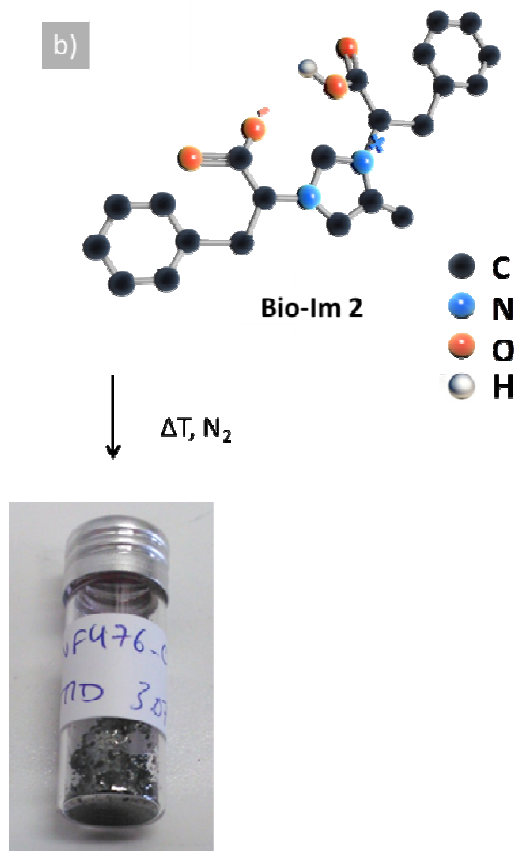

Figure 1 Chemical structures of bio-Ims used as carbon precursors a) bio-Im 1, b) bio-Im 2. c) bulk nitrogen-doped carbon B1S0-1000 obtained after high temperature condensation.

Directly after the high temperature treatment under inert conditions, silvery, shiny and hard solids are obtained (Figure $\mathbf{1} \mathbf{c}$ ). This already shows the different behavior of this precursor/carbon system as ordinary carbon would be matt black. A closer look to the actual chemical composition of these materials reveals one of the main reasons for this metallic reflectance which is the high nitrogen content (Table 1). 
Table 1 Surface areas, yield and chemical composition derived from combustion elemental analysis of the carbons BxSy-Z (B: bio-imidazolium, x: type of bio-imidazolium 1 or 2, S: salt, y: type of salt and Z: synthesis temperature).

\begin{tabular}{|c|c|c|c|c|c|c|}
\hline Name & $\begin{array}{c}\text { BET SSA } \\
\left(\mathbf{m}^{2} / \mathbf{g}\right)\end{array}$ & Yield (\%) & $\mathbf{N}(w t \%)$ & $\mathbf{H}(w t \%)$ & $\mathbf{C}(w t \%)$ & $\mathbf{C / N}$ \\
\hline B1S1-500 & 290 & 76 & 15.85 & 3.077 & 48.24 & 3.0432 \\
\hline B1S2-500 & 388 & 89 & 15.95 & 3.147 & 48.37 & 3.0332 \\
\hline B1S3-500 & 132 & 92 & 17.05 & 3.484 & 49.27 & 2.8903 \\
\hline B2S1-500 & 866 & 33 & 8.55 & 2.754 & 68.59 & 8.0247 \\
\hline B2S2-500 & 388 & 36 & 9.26 & 3.021 & 66.83 & 7.2197 \\
\hline B2S3-500 & 132 & 32 & 9.98 & 3.122 & 59.92 & 6.0056 \\
\hline B1S0-800 & 0 & 15 & 11.65 & 1.587 & 75.26 & 6.4985 \\
\hline B1S1-800 & 983 & 43 & 11.77 & 2.307 & 64.45 & 5.4758 \\
\hline B1S2-800 & 1540 & 49 & 11.09 & 2.264 & 63.01 & 5.6823 \\
\hline B1S3-800 & 1916 & 49 & 11.20 & 2.406 & 61.11 & 5.4558 \\
\hline B1S4-800 & 884 & 13 & 8.97 & 3.245 & 73.80 & 8.2266 \\
\hline B1S0-1000 & 0 & 10 & 6.3 & 1.0 & 90.1 & 14.4 \\
\hline B1S3-1000 & 1596 & 40 & 4.4 & 2.1 & 73.1 & 16.4 \\
\hline
\end{tabular}

With respect to the successful incorporation of high amounts of nitrogen into the carbon lattice, the used amino acids influence the overall heteroatom-content. With the glycine residue being present as the $\mathrm{N}$-substituent on the imidazolium (B1), the nitrogen content in the final carbon is in average twice as high as in the case of the phenylalanine (B2, Table 1). Taking into account the yields found for the two bio-Im carbons, it is unlikely that this is due to the higher carbon content of B2 as otherwise the yield for B2-based carbons must have been higher. It has to be mentioned that the salt, however, has a positive influence on the carbon yield as it drastically increases the amount of the final product. In the case of B2 this is even the key of the synthesis, as without any salt the residues are very low. This phenomenon is still not fully understood, yet it is claimed that the increased ionic interactions, the "solvent-character" of the salt at elevated temperatures as well as the confinement play crucial roles. 
Finally, the nitrogen content also does not drastically depend on the template salt, which suggests that the salt is mainly influencing the porosity, without affecting the chemical composition of the carbon too much.

Scanning electron microscopy (SEM, Figure $\mathbf{2}$ a, b) reveal the typical sponge-like, interconnected morphology of the nitrogen-doped carbons synthesized in the presence of salts, here representatively shown for B1S3-1000 (for more images see Figure SI-1 in the supporting information). The bio-Im-derived carbon morphology is very characteristic for a spinodal microphase demixing of the precursor and salt during the synthesis, more pronounced and clean in structure than in the case of classical IL salt templating. ${ }^{[13]}$ This process for the different salts is schematically depicted in (Figure $\mathbf{2} \mathbf{e - g}$ ).

The homogeneous and light weight structure is further visible by transmission electron microscopy (TEM, Figure 2 c) where thin carbon layers are found. From X-ray diffraction (XRD, Figure $2 \mathrm{~d}$ ) it can be derived that the high temperature materials are not ordinary graphitic carbons but possess only very small stacking heights which supports the high surface areas obtained in nitrogen sorption (Figure 3). A comparison of materials synthesized from B1 without and with different salts, respectively, reveals an ordered nature of carbons already at $800{ }^{\circ} \mathrm{C}$ (Figure SI-2). Here, the carbon B1S0-800 synthesized without salt reveals the most intense XRD peaks which are due to its compact structure. However, even for the highly porous materials B1SY-800 made in the presence of one of the four salts, the diffractogram still shows the typical reflections. 

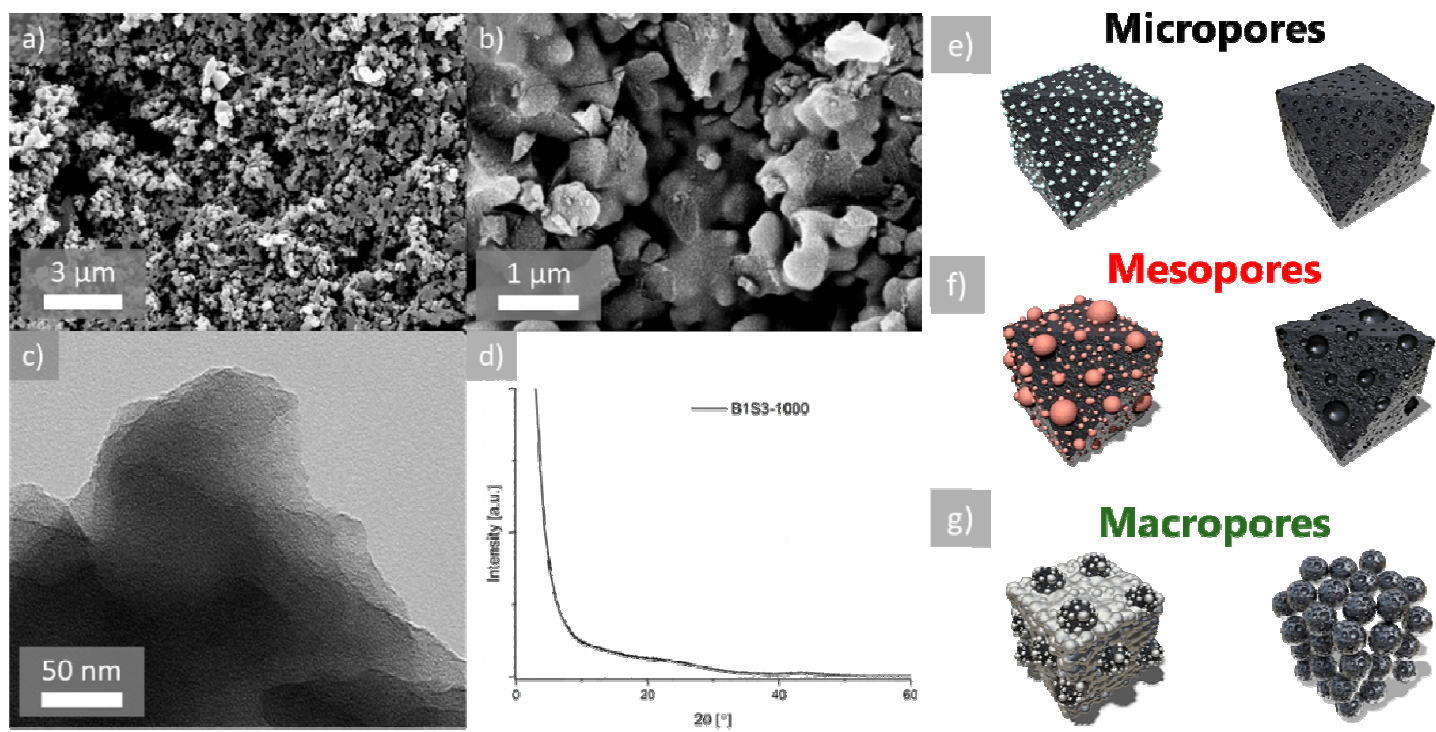

Figure 2 Characterization of B1S3-1000: a), b) SEM, c) TEM and d) XRD, e-g) Illustration of the carbon formation in the presence of three different salt mixtures, e) $\mathrm{LiCl} / \mathrm{ZnCl} 2$ (salt 1=S1), f) $\mathrm{NaCl} / \mathrm{ZnCl}_{2}$ (salt 1=S2) and g) $\mathrm{KCl} / \mathrm{ZnCl}_{2}$ (salt 1=S3), ratio precursor to salt = 1:3.

In order to gain quantitative information about the synthesized mesostructures, nitrogen sorption measurements were performed. In Figure $\mathbf{3}$ the adsorption/desorption isotherm and pore size distribution are shown for the series of B1SX-800 for all four template salts used. As can be already seen from the isotherms, most pores fill-up in the lower relative pressure range which very favorably hints to supermicroporous carbons. Furthermore, especially the B1S4-800 carbon sample displays the combined characteristics of type I and IV isotherms, indicating the coexistence of micro- and mesopores. Additionally, a steep increase in the high relative pressure range, implying a surface adsorption from macropores in the samples is observed. This is in accordance with the morphology observed in the SEM where the interstitial void space of the sponge-like structure contributes the larger pore range. The pore size distribution derived using NLDFT equation gives a range of the pores with diameters between 1.2 and $2.4 \mathrm{~nm}$ and a total pore volume of up to $0.69 \mathrm{cc} / \mathrm{g}$. Nitrogen-doped carbons derived from bio-Ims are therefore high surface area materials with tunable and even hierarchical porosity which is generally of essential interest for applications where small pores but at the same time fast transport is needed. 

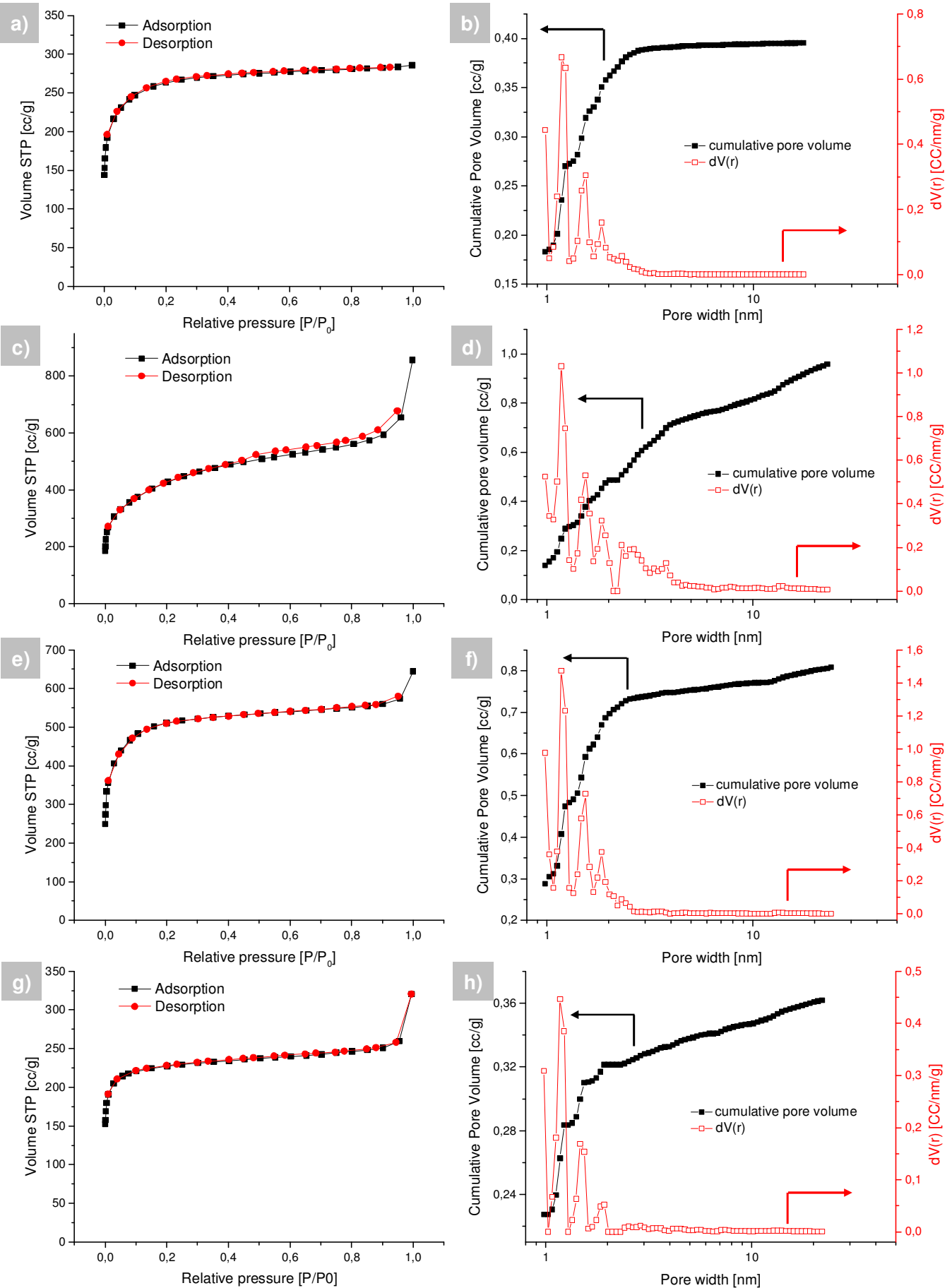

Figure 3 Nitrogen sorption measurements of B1SX-800: a) isotherm, b) pore size distribution of B1S1-800, c), isotherm, d) pore size distribution of B1S2-800, e) isotherm, f) pore size distribution of B1S3-800, g) isotherm, h) pore size distribution of B1S4-800. 
Especially for energy storage applications, such as in supercapacitors, even higher surface areas are wanted. Therefore, the quest for "record surface area carbons" is a very popular topic, yet here the choice of the precursor becomes more and more a combinatorial task together with the right processing technique. In order to test the suitability of the bio-Im precursor also in this direction, we applied several attempts along the salt route. $\mathrm{ZnCl}_{2}$-based salt mixtures could not exceed the value of $1596 \mathrm{~m}^{2} \mathrm{~g}^{-1}$ (Table 1). For this reason, we decided to employ cesium acetate, a low melting point salt, which should guarantee a homogeneous reaction mixture and serve as molecular template due to the very large and polarizable cesium ion. ${ }^{[14]}$ Exemplarily, this is shown for B1 using cesium acetate and the bio-Im 1 in a 1 : 9 mass ratio (Figure SI-3). As for the morphology, TEM (Figure SI-3 a) and SEM pictures (Figure SI-3 b) indicate a structure with winded and fluffy layers which is very different from the carbons obtained from the eutectic mixtures described above. This is supported by XRD measurements which reveal an almost flat diffractogram, indicating a material without graphitic stacks (Figure SI-4). The nitrogen sorption measurement of this material indeed shows an ultrahigh surface area of $2658 \mathrm{~m}^{2} \mathrm{~g}^{-1}$ (Figure SI-3 c, d) which exceeds by far the value for few-layer graphene and even the theoretical value for single-layer graphene.

Recently, we also described the synthesis of highly extended, sheet-like carbons with heteroatom-doping and few nm-thickness, however from glucose. ${ }^{[15]}$ To evaluate a possible generalization of this approach, a $\mathrm{ZnCl}_{2}$-free eutectic mixture was chosen as a solvent in order to work at higher temperature and concentrations otherwise incompatible with $\mathrm{ZnCl}_{2}$. 

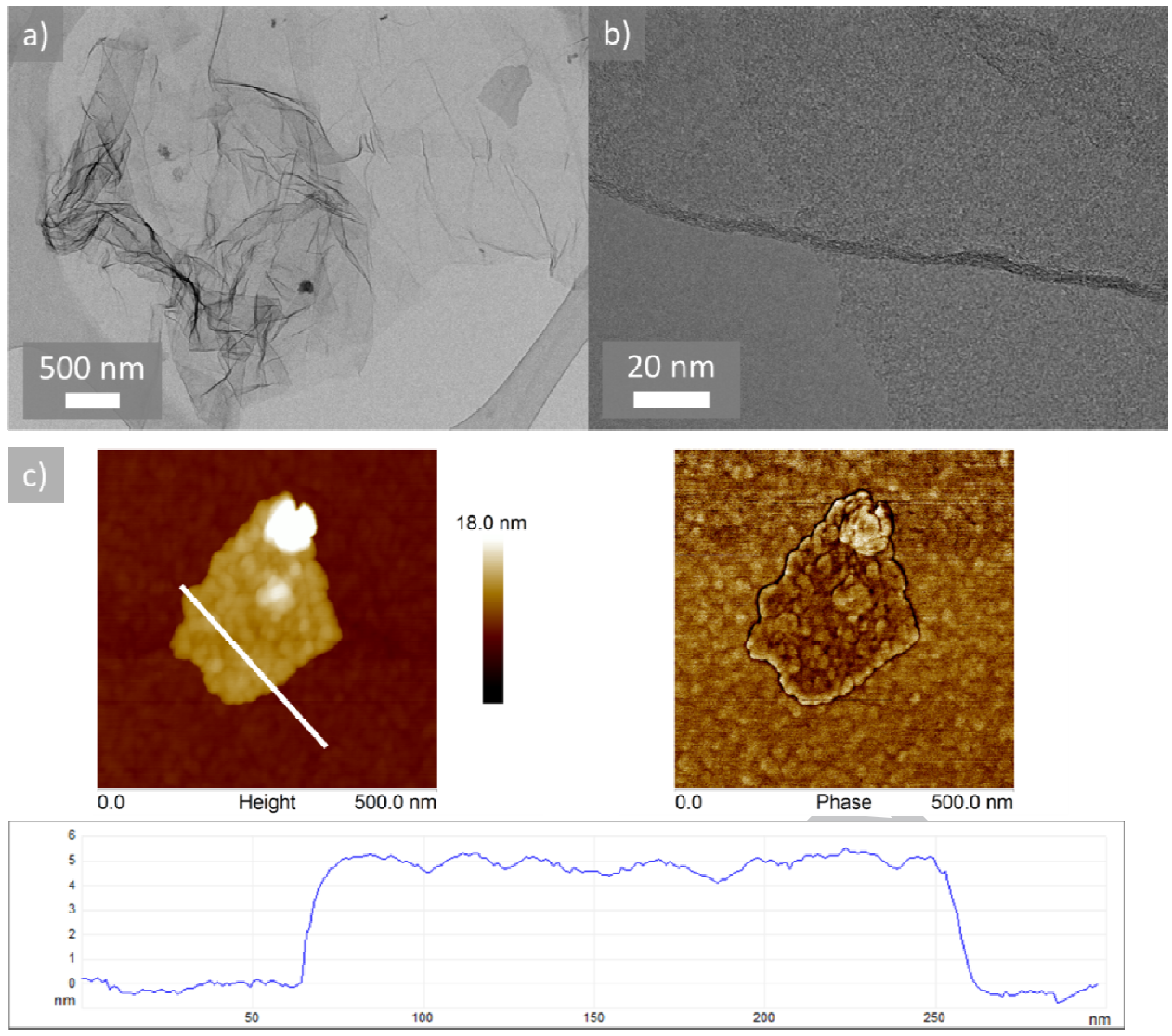

Figure 4 a) HRTEM images of B1S4-800 at very high salt ratio (1:100), b) higher magnification, c) AFM measurements of B1S4-800 at very high salt ratio (1:100).

The carbon derived from B1 in combination with S4 in a weight ratio of B1 to salt of 1:100 indeed result in extended, sheet-like materials as can be clearly seen from the high resolution TEM pictures (Figure $4 \mathbf{a}, \mathbf{b}$ ). The strict 2D-character is further supported by AFM measurements which reveal a highly uniform sheet thickness of about $5 \mathrm{~nm}$ (Figure $4 \mathrm{c}$ ). It is to be noted, that this sheet-like carbon still contains a high nitrogen amount of $7.3 \mathrm{wt} \%$ and possesses a surface area of $763 \mathrm{~m}^{2} \mathrm{~g}^{-1}$ (Figure SI-5) which is a very advantageous factor e.g. for energy-related application.

In conclusion, we presented an alternative approach towards the cheap and scalable synthesis of nitrogen-doped carbon materials of diverse morphology. Compared to standard approaches, the precursors used in this study are "bio-imidazolium zwitterions", which were synthesized from natural amino acids and dioxo-derivatives. Such compounds, act as masked ionic liquids and showed an analogous behavior during carbonization to classical ionic 
liquids. On the one hand, this reduces costs drastically and makes such precursors attractive for functional carbons. On the other hand, the variety of available bio-Ims and the precise incorporation of functionality allow speculating about systematic and rational tuning of the final carbon properties. Thus, the bio-Im platform offers the possibility for low-cost "designer-carbons" which will also allow for the deeper study of the influence of the precursor structure on the final material and thus custom-made carbon materials. Furthermore, these carbons could be shaped to various architectures with controlled porosities via salt templating, using the good and adjustable miscibility with salts. Exemplarily, the pore size was shown to be adjustable from the supermicropore to mesopore range, very high surface areas of $2658 \mathrm{~m} 2 / \mathrm{g}$ could be realized and graphene-like sheet-morphologies are easily synthesized in higher dilution of reactants. As an outlook, it is imaginable that functionality will not only be adjustable through templating and processing, but also by rational insertion of structural motifs, e.g. chirality, which should prevail at least for the carbonaceous intermediates.

\section{References}

[1] H.-P. Cong, J.-F. Chena, S.-H. Yu, Graphene-based macroscopic assemblies and architectures: an emerging material system, Chem Soc Rev. 2014, 43, 7295.

[2] W. Li, Q. Yue, Y. Deng, D. Zhao, Ordered Mesoporous Materials Based on Interfacial Assembly and Engineering, Adv. Mater. 2013, 25, 5129.

[3] S. Zhang, S. Tsuzuki, K. Ueno, K. Dokko, M. Watanabe, Upper Limit of Nitrogen Content in Carbon Materials, Angew. Chem. Int. Ed. 2014, 53, 1.

[4] Y. Zheng, Y. Jiao, Y. Zhu, L. H. Li, Y. Han, Y. Chen, A. Du, M. Jaroniec, S. Z. Qiao, Hydrogen evolution by a metal-free electrocatalyst, Nat. Commun. 2013, 5, 1.

[5] L. Zhao, Z. Bacsik, N. Hedin, W. Wei, Y. Sun, M. Antonietti, M.-M. Titirici, Carbon Dioxide Capture on Amine-Rich Carbonaceous Materials Derived from Glucose, ChemSusChem 2010, 3, 840.

[6] Y. Gong, H. Wang, Z. Wei, L. Xie, Y. Wang, An Efficient Way To Introduce Hierarchical Structure into Biomass- Based Hydrothermal Carbonaceous Materials, ACS Sustainable Chem. Eng. 2014, 2, 2435.

[7] J. Peter Paraknowitsch, J. Zhang, D. Su, A. Thomas, M. Antonietti, Ionic Liquids as Precursors for Nitrogen-Doped Graphitic Carbon, Adv. Mater. 2010, 22, 87. 
[8] J. S. Lee, X. Wang, H. Luo, G. A. Baker, S. Dai, Facile lonothermal Synthesis of Microporous and Mesoporous Carbons from Task Specific lonic Liquids, J. Am. Chem. Soc. 2009, 131, 4596.

[9] S. Zhang, K. Dokko, M. Watanabe, Mater. Horiz. 2015,2, 168

[10] S. Zhang, M. S. Miran, A. Ikoma, K. Dokko, M. Watanabe, J. Am. Chem. Soc. 2014, 136, 1690.

[11] D. Esposito, S. Kirchhecker, M. Antonietti, A Sustainable Route towards Imidazolium Building Blocks Based on Biomass Molecules, Chem. Eur. J. 2013, 19, 15097.

[12] S. Kirchhecker, M. Antonietti, D. Esposito, Hydrothermal decarboxylation of amino acid derived imidazolium zwitterions: a sustainable approach towards ionic liquids, Green Chem., 2014, 16, 3705.

[13] N. Fechler, T.-P. Fellinger, M. Antonietti, "Salt Templating": A Simple and Sustainable Pathway toward Highly Porous Functional Carbons from Ionic Liquids, Adv. Mater. 2013, 25, 75.

[14] K. K. Chung, N. Fechler, M. Antonietti, A Salt-Flux Synthesis of Highly Porous, N- and O-Doped Carbons from a Polymer Precursor and Its Use for High Capacity/High Rate Supercapacitors, Adv. Porous Mater. 2014, 2, 61.

[15] X. Liu , C. Giordano , M. Antonietti, A Facile Molten-Salt Route to Graphene Synthesis, Small 2014, 10, 193. 


\title{
Supporting Information
}

\section{A Sustainable Synthesis Alternative for IL-derived N-doped Carbons: Bio-based-Imidazolium Compounds}

\author{
Seung Jae Yang, Regina Rothe, Sarah Kirchhecker, Davide Esposito, Markus Antonietti, Nina Fechler* \\ Max Planck Institute of Colloids and Interfaces, Department of Colloid Chemistry, MPI Campus Golm, \\ Am Mühlenberg 1, 14476 Potsdam, Germany \\ E-mail: Nina.Fechler@mpikg.mpg.de
}

\section{Materials and Characterization:}

Materials:

Glycine (99.7 \%) was obtained from Merck, L-phenylalanine (cellpure, $\geq 99.0 \%$ ) was purchased from Carl Roth, lithium chloride ( $\mathrm{LiCl}$; 99\%), sodium chloride ( $\mathrm{NaCl}$; 99\%), potassium chloride $(\mathrm{KCl} ; 99 \%)$, zinc chloride $\left(\mathrm{ZnCl}_{2} ; 99 \%\right)$, cesium acetate (CsAc; 99.9\%), methylglyoxal (40\% aqueous solution) and acetic acid ( $\geq 99.7 \%$ ) were obtained from Sigma Aldrich. N-Methyl-2-pyrrolidone (NMP; 99\%) was purchased from Alfa Aesar and formaldehyde ( $37 \%$ aq solution) from Applichem.

Synthesis:

Bio-imidazoliums:

Bio-Im 1 (B1) and Bio-Im 2 (B2) were prepared according to the methods reported in reference [15]. In a typical synthesis of the bio-imidazolium compounds, stoichiometric amounts of amino acid $(0.2 \mathrm{~mol})$, methylglyoxal $(0.1 \mathrm{~mol})$ and formaldehyde $(0.1 \mathrm{~mol})$, were combined in $200 \mathrm{~mL}$ Millipore water in the presence of acetic acid as a catalyst $(0.6 \mathrm{~mol})$. The reaction was allowed to proceed for $1 \mathrm{~h}$ at room temperature for the glycine compound $\mathrm{B} 1$ and $3 \mathrm{~h}$ at $50{ }^{\circ} \mathrm{C}$ for phenylalanine-derived B2. B1 was purified by freeze-drying followed by washing with acetic acid, B2 by extraction with chloroform. 


\section{Bio-imidazolium derived Carbons:}

For the synthesis of the carbons, the respective bio-Im was placed in a ceramic crucible and heated in nitrogen atmosphere to temperatures between 500 and $1000{ }^{\circ} \mathrm{C}$ with a heating rate of $2.5 \mathrm{~K} / \mathrm{min}$ and kept at this temperature for $1 \mathrm{~h}$. The porous carbons were synthesized by mixing the bio-Im with eutectic salt mixtures of $\mathrm{LiCl} / \mathrm{ZnCl} 2$ (salt $1=\mathrm{S} 1$ ), $\mathrm{NaCl} / \mathrm{ZnCl}_{2}$ (salt $1=\mathrm{S} 2$ ), $\mathrm{KCl} / \mathrm{ZnCl}_{2}$ (salt $1=\mathrm{S} 3$ ) and $\mathrm{LiCl} / \mathrm{KCl}$ (salt 1=S4), respectively, in the mass ratio of bioIm:salt as 1:3. For the synthesis of the ultrahigh surface area carbon, the bio-Im was mixed with cesium acetate in a ratio of 1:9. For the synthesis of the carbon sheets, bio-Im 1 was mixed with $\mathrm{LiCl} / \mathrm{ZnCl}_{2}$ in the mass ratio of $1: 100$. The precursor/salt mixtures were then heattreated in the same way as described above. After cooling to room temperature the crude materials were ground and washed with water only, in order to remove the template salt followed by drying in a vacuum oven.

The final carbons are denoted as BxSy-Z, where B stands for bio-imidazolium, $x$ for the type of bio-imidazolium, $\mathrm{S}$ is for salt, $\mathrm{y}$ denotes the type of salt and $\mathrm{Z}$ denotes the synthesis temperature. BxS-0 is the non-porous carbon synthesized without salt.

\section{Characterization:}

Wide angle X-ray-patterns were recorded on a Bruker D8 Advance instrument using Cu Karadiation. Bulk elemental composition was determined by combustion analysis using a Vario Micro device. TEM images were obtained on a Zeiss EM $912 \Omega$ instrument and SEM images on a LEO 1550-Gemini instrument after sputtering with platinum. High resolution TEM images were recorded using a double Cs corrected JEOL ARM 200F instrument.

Nitrogen sorption measurements were performed using $\mathrm{N}_{2}$ at $77 \mathrm{~K}$ after degassing the samples at $150{ }^{\circ} \mathrm{C}$ under vacuum for 20 hours with a Quantachrome Quadrasorb SI porosimeter. The surface area was calculated by applying the Brunauer-Emmett-Teller (BET) model to the isotherm data points of the adsorption branch in the relative pressure range $\mathrm{p} / \mathrm{p} 0<0.3$ in the linear region with the best correlation. The pore size distribution was calculated from N2 sorption data using the nonlocal density functional theory (NLDFT) equilibrium model method for slit pores provided by Quantachrome data reduction software QuadraWin Version 5.05.

For AFM measurements, the sample was dispersed in NMP and thereafter drop-casted on a substrate (freshly cleaved mica), and left to dry at room temperature. AFM height and phase 
images were obtained in the soft tapping mode by Dimension 3100 AFM (Veeco/Bruker, USA) controlled by the Nanoscope IIla controller (Digital Instruments/Bruker, USA). Standard silicone tips (Olympus, model OMCL-AC160TS, Japan; BudgetSensors, model Tap300-G, Bulgaria) with radius around $10 \mathrm{~nm}$ were used. Measurements were performed at room temperature, relative humidity of about $40 \%$, in a low-noise acoustic chamber. For the evaluation of the AFM data Gwyddion 2.38 and NanoScope Analysis 1.50 were used.

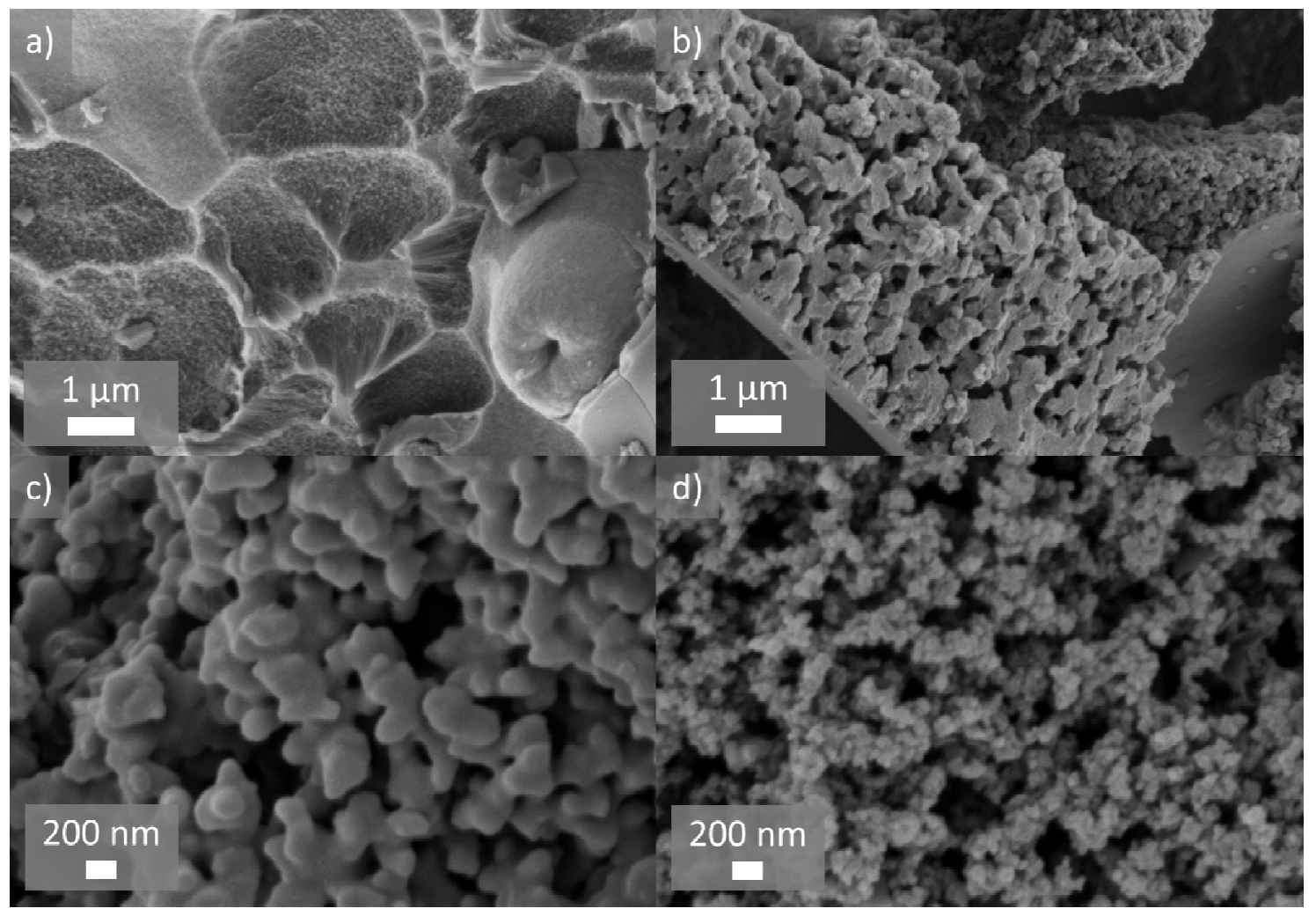

Figure SI-1 SEM of B1SY-800: a) $Y=S 1$, b) $Y=S 2$, c) $Y=S 3$ and d) $Y=S 4$. 


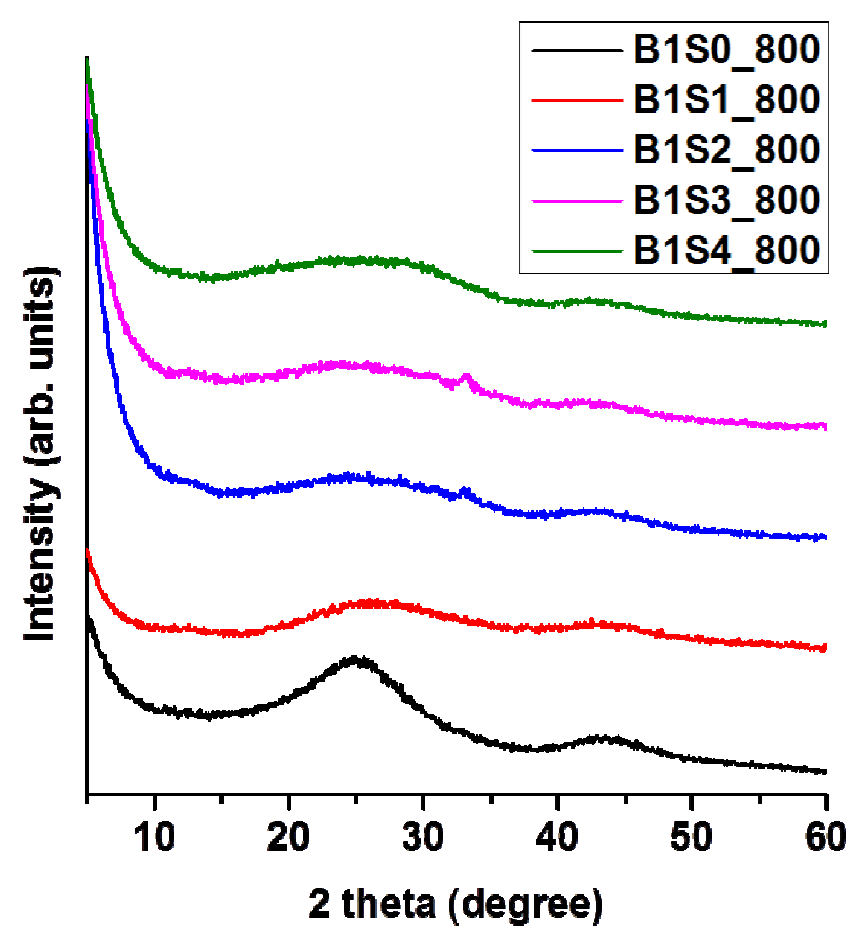

Figure SI-2 XRD of B1SY-800 carbon materials without (S0) and with varying salt templates used (S1S4), mass ratio of bio-Im:salt as 1:3. 

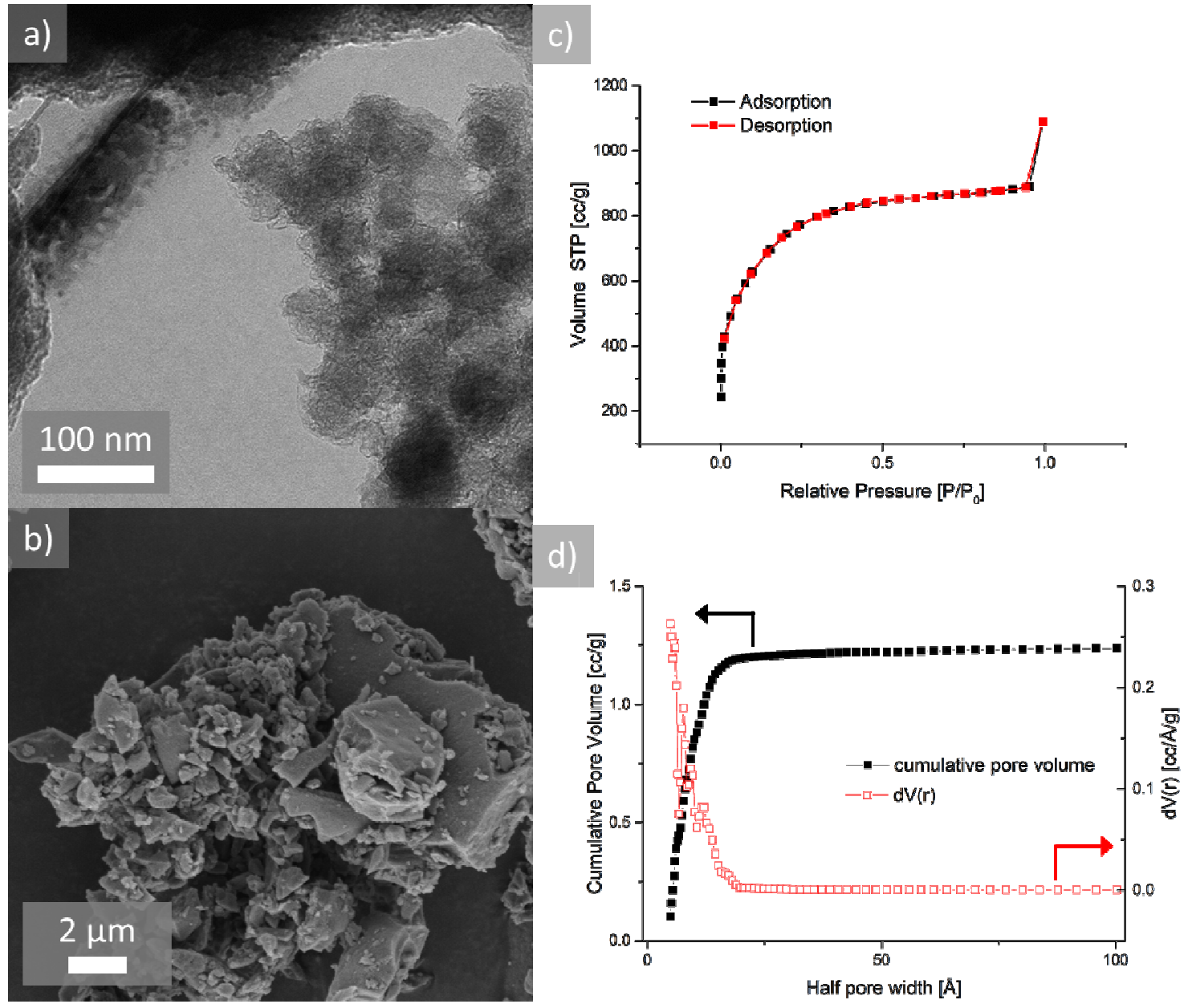

Figure SI-3 a) TEM, b) SEM, c) nitrogen adsorption/desorption isotherm and d) the corresponding pore size distribution of the very high surface area carbon obtained from B1 and cesium acetate at $800^{\circ} \mathrm{C}$. 


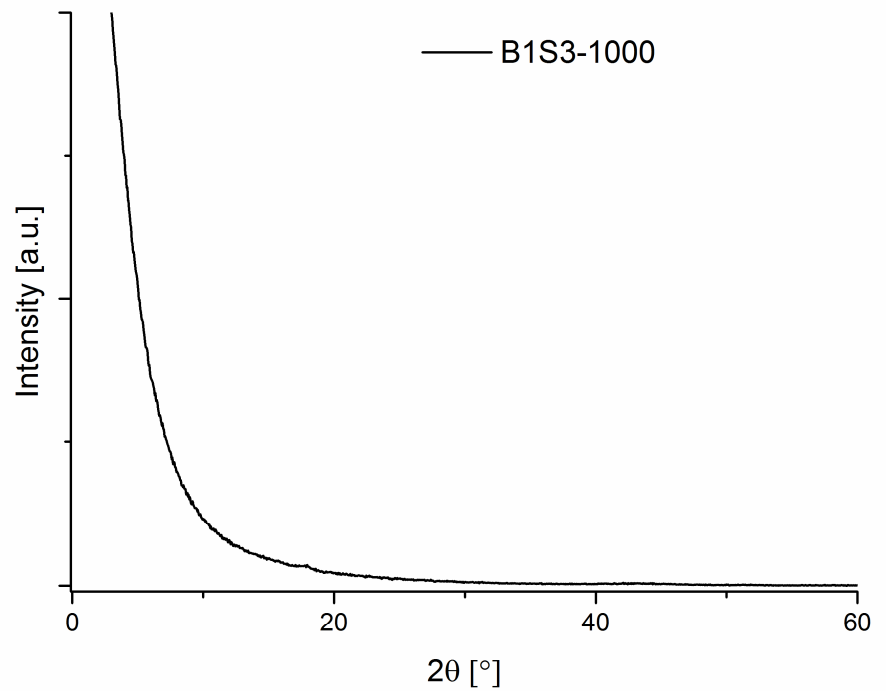

Figure SI-4 XRD pattern of a very high surface area carbon obtained from B1 and cesium acetate at $800^{\circ} \mathrm{C}$.
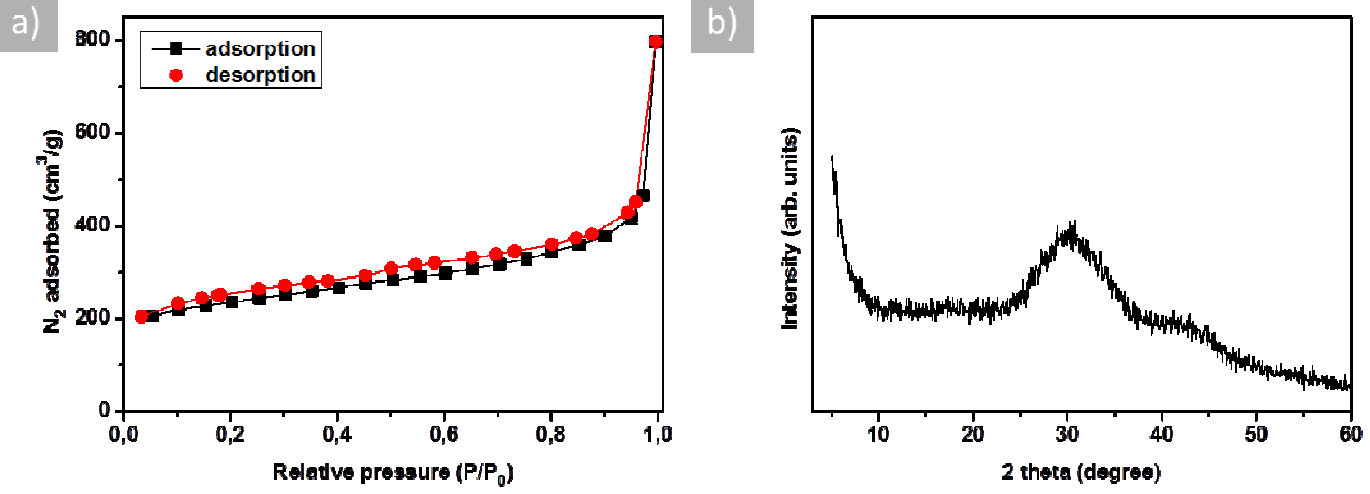

Figure SI-5 a) Nitrogen sorption and b) XRD of B1S4-800 at very high salt ratio (1:100). 